\title{
El proyecto AQUA evalúa el uso eficiente del agua en los jardines de la Edad Moderna
}

El proyecto de investigación AQUA tiene como objetivo evaluar la eficacia de la gestión del agua desde el siglo XVI al XIX en fincas y jardines en Portugal y demostrar que ya se estaban empleando prácticas más sofisticadas en el contexto de granjas y cercas conventuales, convirtiendo los jardines de entonces en laboratorios de innovaciones hidráulicas. Esta gestión del agua se rigió por principios de sostenibilidad asociados a un uso eficiente del agua. La historia lidera este proyecto multidisciplinar que cuenta con la colaboración de historiadores, ingenieros y paisajistas para promover el cruce de conocimientos entre las humanidades y las ciencias.

Ana Duarte Rodrigues | Universidad de Lisboa

Desidério Batista | Universidad del Algarve

URL de la contribución <http://www.iaph.es/revistaph/index.php/revistaph/article/view/5063>

La falta de agua es uno de los mayores problemas de la era del Antropoceno. "Water innovation: Boosting its value for Europe" es un desafío específico del Programa Horizonte 2020. La escasez de agua, su acceso y calidad son tan relevantes hoy como antes. El proyecto HORTO AQUAM SALUTAREM, financiado por la Fundación para la Ciencia y la Tecnología con 219.623,60 euros, para ser desarrollado entre octubre de 2018 y septiembre de 2022, pretende contribuir a este desafío con conocimiento histórico sobre la gestión de sistemas hidráulicos tradicionales y las prácticas sostenibles de regadío.

De cara a los problemas que el mundo enfrenta hoy en día relacionados con el cambio climático $\mathrm{y}$, en consecuencia, la falta de agua y el aumento del proceso de desertificación, hemos planteado la hipótesis de que la gestión del agua en las fincas y cercas conventuales de la Edad Moderna sería eficiente, resiliente y sostenible.

El proyecto de investigación AQUA es desarrollado por un equipo multidisciplinario constituido por historiadores del arte, historiadores de la ciencia, ingenieros hidráuli$\cos y$ arquitectos del paisaje, de distintos países y varias generaciones ${ }^{1}$.

El objetivo del proyecto AQUA es evaluar la eficiencia de la gestión del agua desde el siglo XVI al XIX y establecer las recomendaciones correspondientes sobre la sostenibilidad de los sistemas de energía y agua en jardines y paisajes contemporáneos, que se desdobla en tres objetivos específicos: 1) Contribuir a la historia del agua en la Edad Moderna; 2) Evaluar el manejo y uso eficiente del agua en la Edad Moderna, en términos generales; 3 ) Establecer especificaciones para el ahorro de agua en jardines y paisajes, que permitan el desarrollo de nuevos modelos de uso sostenible del agua.

Para lograr estos objetivos, AQUA se organiza en cuatro ejes conceptuales y metodológicos interrelacionados: 1 ) Estudio de la teoría, accesible en Portugal a través de tratados de hidráulica y gestión del agua; 2) Estudio de manuscritos sobre el agua y sus dispositivos en jardines para analizar cómo se aplicaron los conocimientos teóricos; 3) Trabajo de campo sobre los cuatro casos de estudio seleccionados para su reconstrucción virtual en 3D; 4) Experimentos piloto realizados en el laboratorio del agua para evaluar la eficiencia energética y la sostenibilidad del agua en granjas y cercas conventuales en la Edad Moderna.

La interdisciplinariedad entre la investigación teórica y la aplicada, entre la historia de las ciencias y la tecnología, la ingeniería hidráulica y la arquitectura del paisaje, ha sido la clave del éxito de los resultados de AQUA. Por 

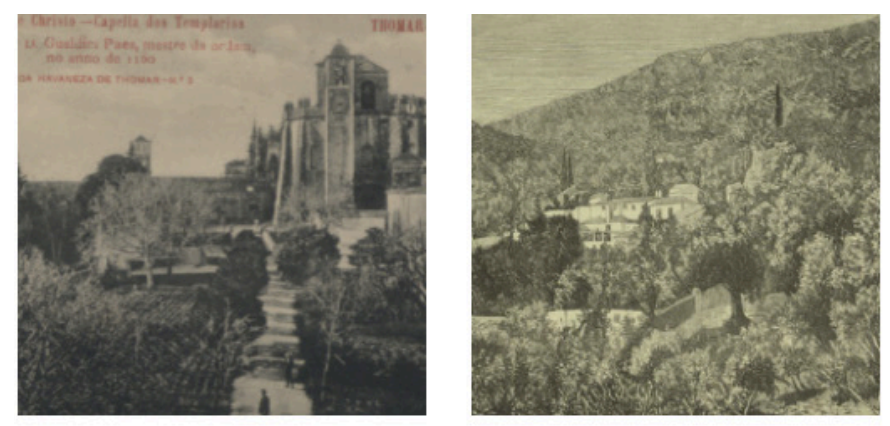

Convent of Christ

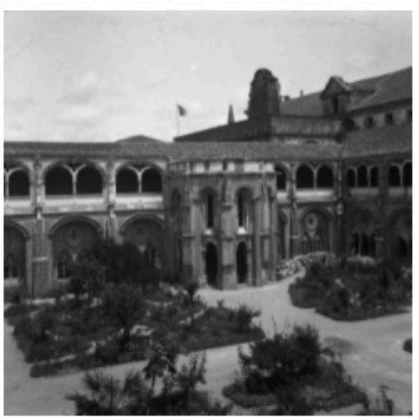

The Monastery of Alcobaça

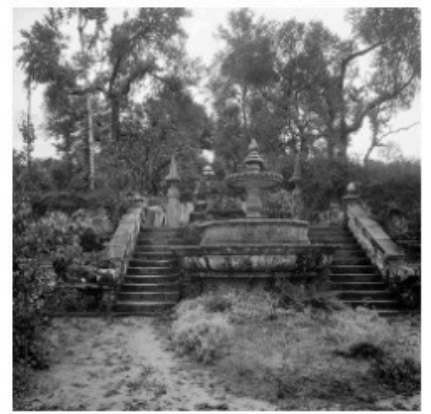

The Monastery of Tibães

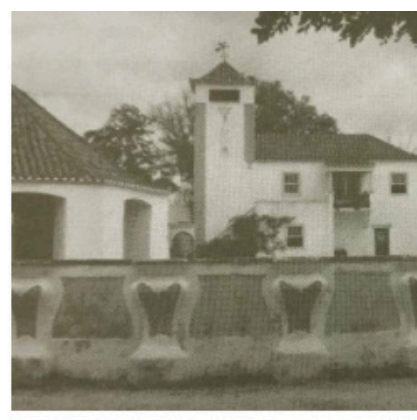

The Villa of the Princess

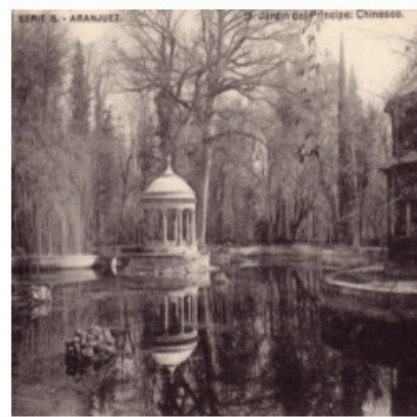

The Royal Estate of Aranjuez

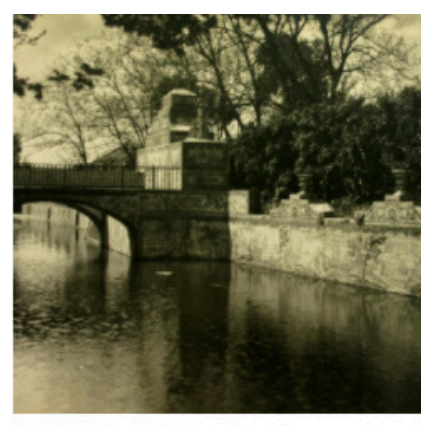

Royal Palace of Queluz

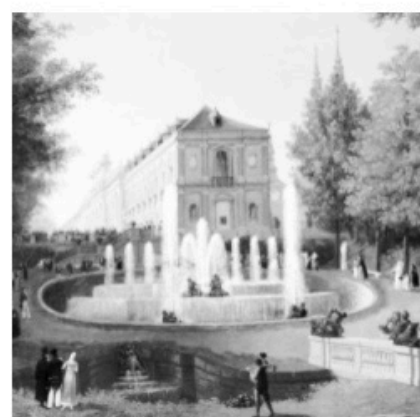

The Royal Estate of San Ildefonso

Casos de estudio del proyecto AQUA

ejemplo, ha permitido cruzar la investigación archivística de fuentes inéditas con el conocimiento conservado en la tratadística, a la vez que se ha complementado con el trabajo de campo realizado sobre los casos de estudio, que incluyó el levantamiento e identificación de estructuras, y la recolección de imágenes, incluyendo vuelos de drones. Además, la investigación histórica sobre la rueda hidráulica de Mouchão, en Tomar, ha alimentado los experimentos piloto que se están llevando a cabo en el Laboratorio del Agua del Instituto Superior Técnico (Lisboa) para, con la reconstitución de la rueda a pequeña escala, calcularse la cantidad de energía por ella producida. Finalmente, este proyecto ha permitido la investigación histórica de bibliografía secundaria y fuentes primarias, incluyendo documentación, iconografía y mapas antiguos, así como visitas y fotografías de restauraciones recientes realizadas en el Palacio Nacional de Queluz, para brindar datos sobre medidas de tubos, fuentes de energía y volumen de depósitos de agua

necesarios para los cálculos y análisis realizados por los ingenieros hidráulicos que evaluaron el consumo de agua en el jardín.

De esta manera, AQUA demostró que los jardines históricos consumían menos agua que los jardines contemporáneos. Al comparar el balance hídrico y energético del Palacio Nacional de Queluz, el parque Carmona en Cascais y un jardín en Vale de Lobos en el Algarve, la ingeniería hidráulica demostró que el uso del agua en el pasado era mucho más parsimonioso y por lo tanto más resistente y sostenible. El sistema funcionaba por gravedad, por lo que el coste energético es mucho menor. En el jardín de Queluz hay sobre todo árboles y arbustos adaptados al clima mediterráneo y sin riego, mientras que en los jardines contemporáneos predominan los céspedes, que requieren recursos hídricos que son escasos y se agotarán en las próximas décadas. Por otro lado, los jardines históricos se beneficiaron de la sabiduría y 
el cuidado de los jardineros que solo regaban los parterres cuando había una necesidad absoluta, a diferencia del riego automático que riega incluso sin ser necesario, con dos consecuencias negativas: el aumento del consumo de agua y el fomento de plantas "adictas" que, por lo tanto, dependen más del riego regular. Finalmente, la escasez de agua en el Mediterráneo, con un verano largo y seco, impuso no solo el mayor almacenamiento posible, sino también una gestión racional del agua, aprovechando y reutilizando toda el agua. El lema que se utiliza a menudo hoy en día para llamar la atención sobre la escasez de agua ("cada gota cuenta") era una realidad en la Península Ibérica hasta hace aproximadamente un siglo.

Otros casos de estudio revelaron el papel de la pericia local en el uso inteligente del agua; en Seixal, por ejemplo, se aprovecharon las condiciones geográficas y biofísicas del lugar para establecer una serie de molinos mareales dedicados a la molienda de harina, cuya demanda aumentó exponencialmente a finales de siglo XV con la necesidad de producir galletas para los Descubrimientos. Por otro lado, tanto el estudio de este caso como el realizado sobre las estructuras hidráulicas instaladas a lo largo del río Nabão, revelaron que ya en el siglo XIX, principios del XX, los ingenieros propusieron lo que hemos hecho en AQUA: que se utilizase este conocimiento del pasado y aprovechase las fuentes de energía limpia adaptando las ruedas hidráulicas a la convivencia con la energía eléctrica. La investigación realizada sobre el sistema hidráulico del Convento de Cristo en Tomar saltó los muros de la cerca y se extendió a lo largo del río Nabão, revelando no solo el poder de la Orden de Cristo sobre todo el territorio, incluido el río, con decisión sobre cada iniciativa, sino mostrando también a través de mapas la evolución de las estructuras hidráulicas en la región desde la Edad Media hasta el siglo $X X$.

En el marco del proyecto AQUA, por primera vez en Portugal, se ha llevado a cabo una documentación de los tratados hidráulicos y de gestión del agua de la Edad Moderna que circulaban en Portugal, creando una base de datos en la pagina web con información no solo de cada uno de estos tratados, sino también de las distin- tas ediciones y traducciones que se realizaron de cada uno de ellos.

Finalmente, pero quizás lo más importante, AQUA se ha constituido como un espacio de formación para una nueva generación de investigadores en estos temas, que hasta hace poco había sido descuidado, posibilitando la conclusión de tres tesis de máster y otras dos en fase finalización, y una beca para un joven investigador de la Universidad de Cagliari para trabajar en el equipo del AQUA.

En cuanto a resultados, destaca la obra de referencia sobre la historia del agua en la Península Ibérica entre los siglos XVI y XIX, The History of Water Management in the Iberian Peninsula between the 16th and 19th cen-
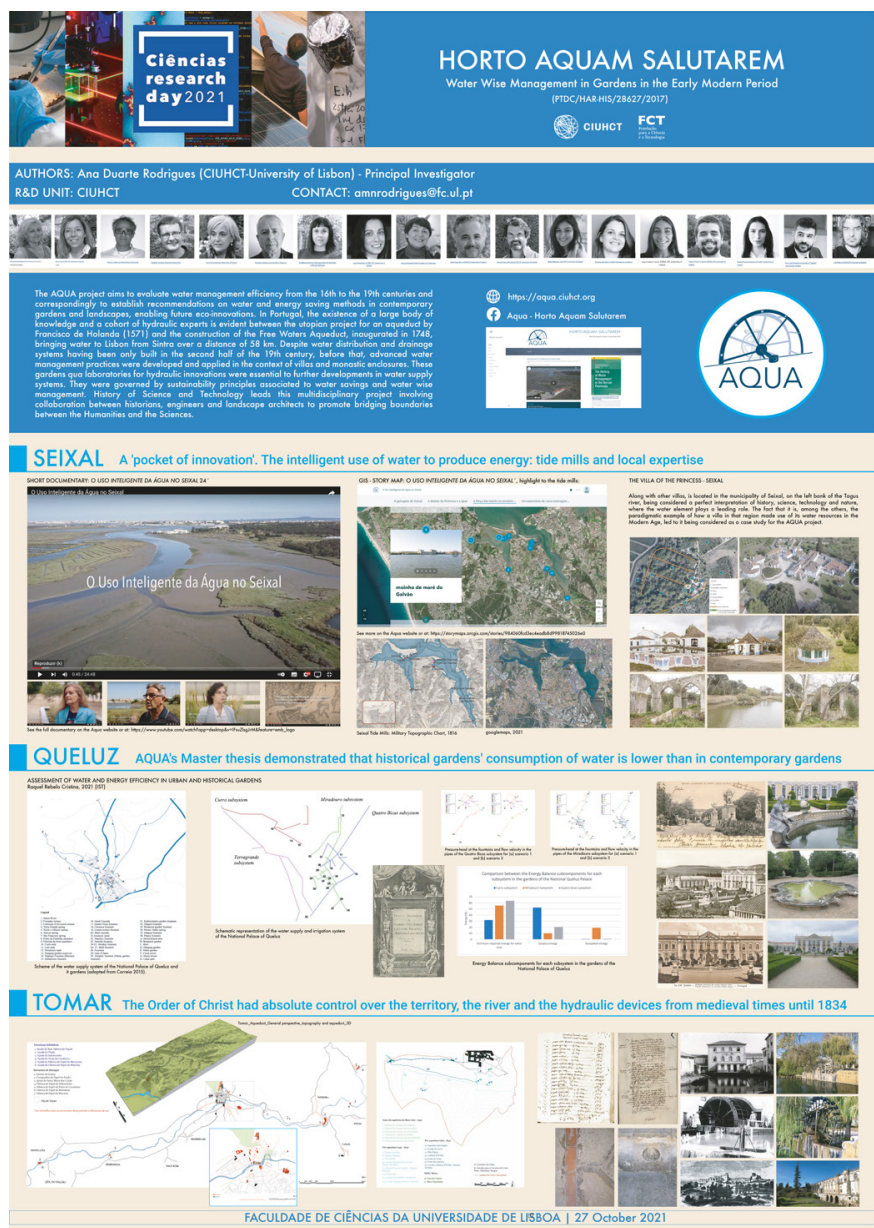
turies, editada por Springer en 2020, que fue capaz de reunir a especialistas nacionales e internacionales para cubrir distintas temáticas, desde el abastecimiento de las ciudades, pasando por las grandes obras de modificación del paisaje, hasta la enseñanza y formación en hidráulica.

\section{NOTAS}

1. Ana Duarte Rodrigues (investigadora principal), historiadora de la ciencia, Centro Interuniversitario de Historia de las Ciencias y de la Tecnología (CIUHCT), Universidad de Lisboa; Dídia Covas (co-investigadora principal), ingeniera hidráulica, Civil Engineering Research and Innovation for Sustainability (CERIS), Instituto Superior Técnico (IST), Universidad de Lisboa; Matteo Valleriani, historiador de la ciencia, Max Planck Institute (Alemania); Anatole Tchikine, historiador del arte, Dumbarton Oaks/Universidad de Harvard (Estados Unidos); Aurora Carapinha, arquitecta del paisaje, Centro de Historia del Arte e Investigación Artística (CHAIA), Universidad de Évora; Desidério Batista, arquitecto del paisaje, CHAIA/ UÉ, Universidad del Algarve; Magdalena Merlos, historiadora del arte, Ayuntamiento del Real Sitio y Villa de Aranjuez (España); Laura Monteiro, ingeniera hidráulica, CERIS, IST/Universidad de Lisboa; Urszula Sowina, historiadora de la ciencia, Academia Polaca de las Ciencias (Polonia); João Puga Alves, arquitecto, doctorando en Historia y Filosofía de las Ciencias, CIUHCT, Universidad de Lisboa; Ignacio García-Pereda, historiador de la ciencia, CIUHCT, Universidad de Lisboa; Clara Marques y Patrícia Monteiro, becarios de investigación del AQUA, CIUHCT, Universidad de Lisboa; Raquel Cristina y Miguel Capelo, becarios de investigación del AQUA, CERIS, IST/Universidad de Lisboa; Paula Medeiros, becaria de investigación del AQUA, Universidad del Algarve; Pier Luigi Pireddu, becario ERASMUS, Universidad de Cagliari (Italia); Luís Ribeiro, historiador de la ciencia, CIUHCT, Universidad de Lisboa. 